\title{
Chlorine Vacancy Passivation in Mixed-Halide Perovskite Quantum Dots by Organic Pseudohalides Enables Efficient Rec. 2020 Blue Light-Emitting Diodes
}

Xiaopeng Zheng1,\|, Shuai Yuan2,", Jiakai Liu1,", Jun Yin1,", Fanglong Yuan 3,4," Wan-Shan Shen², Kexin $\mathrm{Yao}^{1}$, Mingyang $\mathrm{Wei}^{3}$, Chun Zhou ${ }^{3}$, Kepeng Song ${ }^{1}$, Bin-Bin Zhang ${ }^{5}$, Yuanbao Lin ${ }^{1}$, Mohamed Nejib Hedhili $^{6}$, Nimer Wehbe ${ }^{6}$, Yu Han ${ }^{1}$, Hong-Tao Sun ${ }^{5}$, Zheng-Hong Lu ${ }^{4}$, Thomas D. Anthopoulos ${ }^{1}$, Omar F. Mohammed ${ }^{1}$, Edward H. Sargent ${ }^{3}$, Liang-Sheng Liao ${ }^{2, *}$, and Osman M. Bakr ${ }^{1, *}$

${ }^{1}$ Division of Physical Sciences and Engineering, King Abdullah University of Science and Technology, Thuwal 23955-6900, Saudi Arabia.

${ }^{2}$ Jiangsu Key Laboratory for Carbon-Based Functional Materials \& Devices, Institute of Functional Nano \& Soft Materials (FUNSOM), Soochow University, Suzhou, Jiangsu 215123, China.

${ }^{3}$ Department of Electrical and Computer Engineering, University of Toronto, 35 St George Street, Toronto, Ontario M5S 3G4, Canada.

${ }^{4}$ Department of Materials Science and Engineering, University of Toronto, 184 College Street, Toronto, Ontario M5S 3E4, Canada.

${ }^{5}$ College of Chemistry, Chemical Engineering and Materials Science, Soochow University, Suzhou 215123, China.

${ }^{6}$ Imaging and Characterization Core Lab, King Abdullah University of Science and Technology, Thuwal 23955-6900, Saudi Arabia.

\begin{abstract}
Blue-emitting perovskites are easily attainable by precisely tuning the halide ratio of mixed halide $(\mathrm{Br} / \mathrm{Cl})$ perovskites (MHPs). However, the adjustable halide ratio also hinders the passivation of $\mathrm{Cl}$ vacancies - the main source of trap states leading to inferior performance blue MHP light-emitting diodes (LEDs). Here, we report a strategy to passivate $\mathrm{Cl}$ vacancies in MHP quantum dots (QDs) using non-polar-solvent-soluble organic pseudohalide (n-dodecylammonium thiocyanate (DAT)), enabling blue MHP LEDs with greatly enhanced efficiency. Density-functionaltheory calculations reveal that the thiocyanate $\left(\mathrm{SCN}^{-}\right)$groups fill in the $\mathrm{Cl}$ vacancies and remove electron traps within the bandgap. DAT-treated $\mathrm{CsPb}\left(\mathrm{Br}_{\mathrm{x}} \mathrm{Cl}_{1-\mathrm{x}}\right)_{3}$ QDs exhibit near unity ( 100\%) photoluminescence quantum yields; and their blue $(\sim 470 \mathrm{~nm})$ LEDs are spectrally stable with an external quantum efficiency (EQE) of $6.3 \%$ - a record for perovskite LEDs emitting in the $460-480 \mathrm{~nm}$ range relevant to Rec. 2020 display standards - and a half-lifetime of $\sim 99 \mathrm{~s}$.
\end{abstract}

Perovskite semiconductors are attracting widespread attention as an emerging class of emitters for light-emitting diodes (LEDs), due to their facile processing, tunable emission, and high photoluminescence quantum yield (PLQY). While green and red perovskite LEDs have advanced at a rapid pace, achieving quantum efficiencies (EQEs) $>20 \%$,

${ }^{3}$ blue perovskite LEDs still lag far behind with EQEs of
$<5 \%$ for $\lambda_{\text {emission }}$ at $460-480 \mathrm{~nm}$ (the wavelength of the Rec. 2020 is $467 \mathrm{~nm})^{4-7}$ and $<11 \%$ for $\lambda_{\text {emission }}$ at $480-490 \mathrm{~nm}^{8-10}$, i.e. sky blue (Table 1). Among the perovskite blue-emitters, mixed halide $(\mathrm{Cl} / \mathrm{Br})$ perovskites (MHPs) afford an easily tunable emission spectra in the blue by adjusting the halide $(\mathrm{Cl} / \mathrm{Br})$ ratio. ${ }^{11-15}$ However, despite being the facile approach to yield perovskite blue LEDs that emit in the color range relevant to display standards (Rec. 2020), blue MHP LEDs are still in their infancy, and exhibit both poor efficiency and short operational half-life. ${ }^{9}$

Halogen vacancies are the predominant defect species in inorganic cesium lead halide $\left(\mathrm{CsPbX}_{3}, \mathrm{X}=\mathrm{Cl}, \mathrm{Br}, \mathrm{I}\right)$ QDs. ${ }^{16-17}$ However, unlike "defect-tolerant" $\mathrm{CsPbI}_{3}$ and $\mathrm{CsPbBr}_{3}$, chlorine vacancies in MHP $\mathrm{CsPb}\left(\mathrm{Br}_{\mathrm{x}} \mathrm{Cl}_{1-\mathrm{x}}\right)_{3}$ create relatively deeper trap states within the bandgap that irreversibly capture charge carriers and dramatically suppress the radiative recombination. ${ }^{18-25}$ Moreover, these defects initiate and catalyze device degradation by facilitating rapid ion migration and making the perovskite more vulnerable to external stimuli under atmospheric and operational conditions. ${ }^{20,}$ 26-27 Therefore, the suppression of $\mathrm{Cl}$ vacancies is a prerequisite for achieving efficient and stable blue MHP LEDs. 
Table 1. Summary of Key Parameters for Blue Perovskite LEDs

\begin{tabular}{|c|c|c|c|c|c|c|}
\hline \multicolumn{2}{|c|}{ Perovskite category and examples } & \multirow{2}{*}{$\begin{array}{c}\text { Emission } \\
\text { peak [nm] } \\
490\end{array}$} & \multirow{2}{*}{$\begin{array}{c}\text { Color } \\
\text { Sky-blue }\end{array}$} & \multirow{2}{*}{$\begin{array}{c}\text { FWHM } \\
{[\mathrm{nm}]} \\
28\end{array}$} & \multirow{2}{*}{$\begin{array}{c}\mathrm{EQE} \\
(\%)\end{array}$} & \multirow{2}{*}{$\begin{array}{c}\text { Reference } \\
8\end{array}$} \\
\hline Pure bromide, quasi-2D & $\mathrm{PEA}_{2}(\mathrm{MACs})_{1.5} \mathrm{~Pb}_{2.5} \mathrm{Br}_{8.5}$ & & & & & \\
\hline Mixed halide, 2D-3D & PEACl:CsPbBr 3 & 485 & Sky-blue & 23 & 11 & 7 \\
\hline Pure bromide, 3D & $\mathrm{Cs}_{\mathrm{x}} \mathrm{FA}_{1-\mathrm{x}} \mathrm{PbBr}_{3}$ & 483 & Sky-blue & 26 & 9.5 & 9 \\
\hline Mixed halide, quasi-2D & $\mathrm{CsPbCl}_{0.9} \mathrm{Br}_{2.1}$ & 480 & Blue & 21 & 5.7 & 10 \\
\hline Mixed halide, 2D-3D & PEACl:CsPbBr 3 & 477 & Blue & 23 & 4.8 & 7 \\
\hline Pure bromide, quasi-2D & $\mathrm{PEA}_{2}\left(\mathrm{Rb}_{0.6} \mathrm{Cs}_{0.4}\right)_{2} \mathrm{~Pb}_{3} \mathrm{Br}_{10}$ & 475 & Blue & 20 & 1.35 & 4 \\
\hline Mixed halide, 3D & $\mathrm{CsMn}_{\mathrm{y}} \mathrm{Pb}_{1-\mathrm{y}} \mathrm{Br}_{\mathrm{x}} \mathrm{Cl}_{3-\mathrm{x}}$ & 466 & Blue & 17.9 & 2.12 & 5 \\
\hline Mixed halide, quasi-2D & $\mathrm{BA}_{2} \mathrm{Cs}_{\mathrm{n}-1} \mathrm{~Pb}_{\mathrm{n}}(\mathrm{Br} / \mathrm{Cl})_{3 \mathrm{n}+1}$ & 465 & Blue & 23 & 2.4 & 6 \\
\hline Mixed halide, 3D & $\mathrm{CsPb}\left(\mathrm{Br}_{\mathrm{x}} \mathrm{Cl}_{1-\mathrm{x}}\right)_{3}$ & 471 & Blue & 17 & 6.3 & This work \\
\hline
\end{tabular}

We sought to rationally identify efficient $\mathrm{Cl}$ vacancy passivating agents for blue-emitting MHPs out of a large pool of potential candidate materials. Organic halides, which are usually useful passivation agents for halogen vacancies in $\mathrm{CsPbBr}_{3}$ and $\mathrm{CsPbI}_{3}$, are incompatible with MHPs because of the unwanted peak shifts that occur during their fast halide exchange with MHPs. Pseudohalogens, on the other hand, such as thiocyanate $\left(\mathrm{SCN}^{-}\right)$, can fill in the halogen vacancies without appreciably changing the emission spectrum. ${ }^{21}$ However, in general, low solubility in non-polar solvents is a major hurdle facing most perovskite passivation agents, including pseudohalogens (polar solvents decompose the highly ionic perovskites). Potentially useful non-polar solvents for passivation processing of MHP emitters should also be halogen-free, as halogen-containing solvents (e.g. chloroform) carry halide ions that may halide-exchange and shift the bandgap of MHP QDs. Therefore, any candidate pseudohalogen passivators for $\mathrm{Cl}$ vacancies in MHP QDs should be soluble in a non-polar solvent that is compatible with MHP QD processing.

With these constraints in mind, we searched for an organic thiocyanate (RSCN) with sufficient hydrophobic groups to dissolve in non-polar, non-halogenated solvents. We found that n-dodecylammonium thiocyanate (DAT; $\mathrm{C}_{12} \mathrm{H}_{25} \mathrm{NH}_{3} \mathrm{SCN}$ ) has a high solubility $(>100 \mathrm{mg} / \mathrm{ml})$ in toluene - a non-polar solvent that is used during MHP QD synthesis and storage; while neither a shorter alkyl chain n-butylammonium thiocyanate (BAT; $\mathrm{C}_{4} \mathrm{H}_{9} \mathrm{NH}_{3} \mathrm{SCN}$ ) nor an equivalently long chained $\mathrm{n}$-dodecylammonium chloride (DAC; $\mathrm{C}_{12} \mathrm{H}_{25} \mathrm{NH}_{3} \mathrm{Cl}$ ) could be dissolved in that solvent (Figure S1). In the following studies, we subjected the MHP QDs to a post-treatment of DAT by mixing them together in toluene (0.01 wt.\% DAT in MHP QD dispersion; see Supporting Information, $\mathrm{SI})$. $\mathrm{CsPb}\left(\mathrm{Br}_{\mathrm{x}} \mathrm{Cl}_{1-\mathrm{x}}\right)_{3}$ QDs were synthesized according to a well-established synthesis protocol. ${ }^{11-15}$, 28

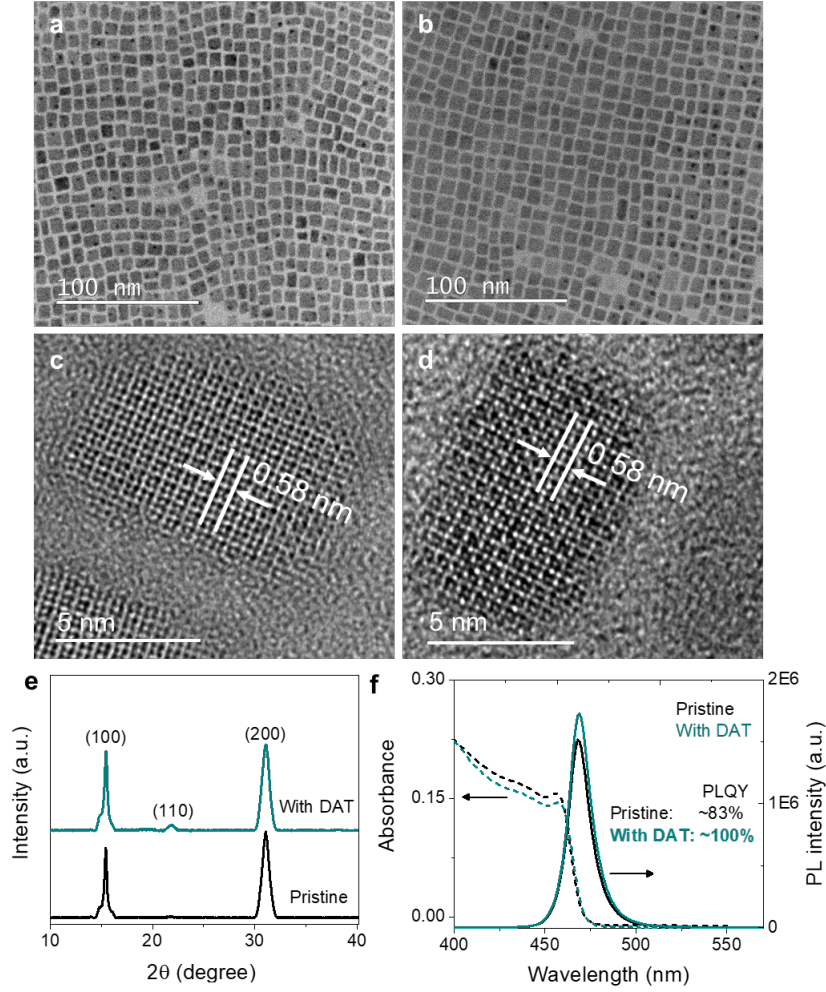

Figure 1. (a, b) Transmission electron microscopy (TEM) images of (a) pristine MHP QDs and (b) DAT-treated MHP QDs, respectively. (c, d) High-resolution TEM (HR-TEM) images of (c) pristine MHP QDs and (d) DAT-treated MHP QDs, respectively. (e) XRD patterns of pristine MHP QDs and DAT-treated MHP QDs. (f) Steady-state optical absorption and PL spectra of pristine MHP QDs and DAT-treated MHP QDs.

The influence of the DAT post-treatment on the structural properties of MHP QDs was investigated by X-ray powder diffraction (XRD) and transmission electron microscopy (TEM). The pristine MHP QDs and DAT-treated MHP QDs were found to have a cuboid shape with average sizes of 8.7 $\mathrm{nm}$ and $9.2 \mathrm{~nm}$, respectively (Figure 1a, b, and Figure S2). Both pristine MHP QDs and DAT-treated MHP QDs have a lattice constant (d) of $0.58 \mathrm{~nm}$, corresponding to the (100) crystal plane of the cubic phase perovskite, ${ }^{14-15}$ indicating 
that the DAT-treatment does not alter the MHP QD's crystal structure (Figure 1c, d). The XRD patterns shows that both pristine MHP QDs and DAT-treated MHP QDs have a cubic phase (Figure 1e). To verify the presence of DAT in the MHP QD films at a high level of sensitivity, we collected secondary ion mass spectrometry (SIMS) depth profiles on drop-casted QD films. ${ }^{29-30}$ The SIMS measurements (Figure S3) show that the signal of sulfur in DAT-treated MHP QDs is of over one order of magnitude higher than that of the pristine QDs.

We observed a notable enhancement of the PL intensity and PLQY of these MHP QDs with DAT-treatment. The pristine MHP QDs exhibit an emission peak at $468.4 \mathrm{~nm}$, with a full width at half maximum (FWHM) of $\sim 15 \mathrm{~nm}$ and a PLQY of $83 \%$. On the other hand, the DAT MHP QDs exhibit an emission peak at $468.8 \mathrm{~nm}$ and a PLQY that reaches to near unity $(\sim 100 \%)$ (Figure 1f). We note that the slight shift of emission peaks can be attributed to the minor change in the NC's size caused by DAT-treatment. ${ }^{13}$

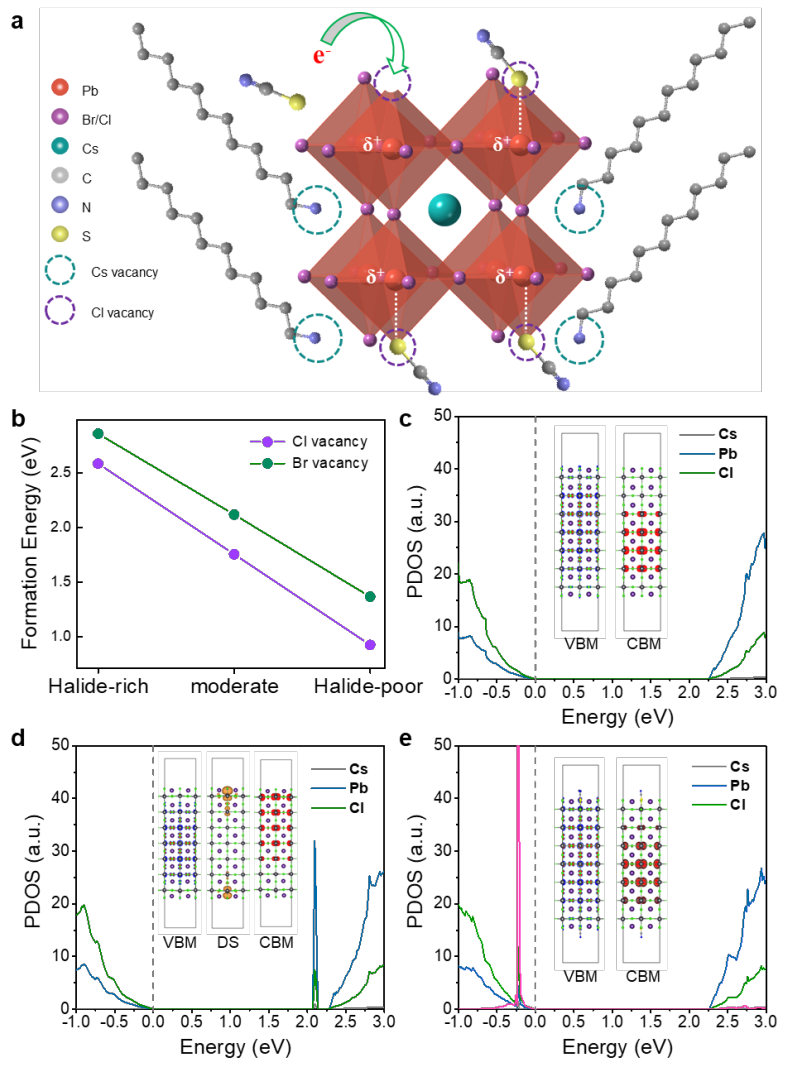

Figure 2. (a) Illustration of $\mathrm{Cl}$ vacancy induced Coulomb trap site formation, electron trapping, and self-assembly of organic thiocyanate (RSCN) on the defect sites in MHP. (b) Calculated defect formation energies of $\mathrm{Cl}$ vacancy on $\mathrm{CsPbCl}_{3}$ (100) surface and $\mathrm{Br}$ vacancy on $\mathrm{CsPbBr}_{3}$ (100) surface at different growth conditions. (c-e) Calculated project density of states (PDOS) and electronic charge densities of valence band maximum (VBM), defect state (DS), and conduction band minimum (CBM) for (c) CsCl-rich CsP$\mathrm{bCl}_{3}$ slab, (d) $\mathrm{CsPbCl}_{3}$ slab with surface $\mathrm{Cl}$ vacancies, and (e) CsP$\mathrm{bCl}_{3}$ slab with surface filled $\mathrm{SCN}^{-}$groups. The pink line shows the contribution from $\mathrm{SCN}^{-}$to DOS. The valence band maximum (VBM) is set at zero energy with a vertical grey dotted line as a guide to the eye.
To further elucidate the mechanism underpinning the PLQY enhancements, we investigated the effect of DAT-treatment on the electronic properties of $\mathrm{CsPCl}_{3}$ by using DFT calculations. We considered three slab models which are $(i)$ an ideal $\mathrm{CsPbCl}_{3}$ slab, (ii) a $\mathrm{CsPbCl}_{3}$ slab with removed surface $\mathrm{Cl}$ atoms, and (iii) a $\mathrm{CsPbCl}_{3}$ slab treated with the $\mathrm{SCN}^{-}$on the surface. Here the $\mathrm{Cl}$ vacancy is the dominant defect on the $\mathrm{CsPbCl}_{3}$ surface as the formation energy of surface $\mathrm{Cl}$ vacancy is even lower than $\mathrm{Br}$ vacancy in $\mathrm{CsPbBr}$, which is known as the major defect species in $\mathrm{CsPbBr}_{3}$ (Figure 2b). ${ }^{19}$ In the ideal $\mathrm{CsPbCl}_{3}$ slab, both hole and electron wavefunctions are delocalized in the bulk (Figure 2c). The $\mathrm{Cl}$ vacancy leads to the formation of a trap state that is $\sim 0.2 \mathrm{eV}$ below the conduction band with a localized charge density on the surface layer (Figure 2d), acting as an electron-trapping center for the nonradiative recombination. Once the $\mathrm{Cl}$ vacancy on the surface is filled by $\mathrm{SCN}^{-}$, it removes the midgap states and enriches the top of the valance band density through contributions from $\mathrm{SCN}^{-}$(Figure 2e). The reduction of the trap states is confirmed by prolonged PL lifetime, deduced from time-resolved photoluminescence decay measurements, for the MHP films after DAT treatment (Figure S4).

We summarized the likely mechanisms behind the PLQY enhancement in Figure 2a. $\mathrm{Cl}$ vacancy formation leads to a net positive charge residing on a $\mathrm{Pb}$ atom, which is also known as an under-coordinated $\mathrm{Pb}$ atom. ${ }^{31}$ The photoexcited electrons can be electrostatically attracted into this Coulomb trap site ( $\mathrm{Cl}$ vacancies). This view is consistent with the observation of picosecond electron trapping as the dominant nonradiative channel impairing the PLQYs of $\mathrm{CsPbCl}_{3}$ $\mathrm{NCs} .{ }^{24} \mathrm{SCN}^{-}$can fill these $\mathrm{Cl}$ vacancies, and donate electron density to under-coordinated $\mathrm{Pb}$ atoms of MHP $\mathrm{QDs}^{32-34}$, resulting in the removal of electron traps. The co-existence of two chemical environments ( $\mathrm{Pb}$-halide and $\mathrm{Pb}-\mathrm{SCN}$ ) as demonstrated in a previous study, indicates $\mathrm{SCN}^{-}$'s capability of filling halide vacancies, ${ }^{35}$ which is in good agreement with the proposed mechanisms underpinning the $\mathrm{Cl}$ vacancy passivation enabled by $\mathrm{SCN}^{-}$.

Encouraged by the improved PLQYs, we fabricated LEDs using these MHP QD inks with the DAT added. LEDs were structured as: indium tin oxide (ITO) glass substrate/Poly(9,9-dioctylfluorene-alt-N-(4-sec-butylphenyl)diphenylamine) (TFB)/nafion perfluorinated resin (PFI)/MHP QDs/tris(2,4,6-triMethyl-3-(pyridin-3-yl)phenyl)borane (3TPYMB)/Liq/Al, as shown in Figure 3a. The cross-sectional high-angle annular dark-field scanning transmission electron microscopy (HAADF-STEM) image of the MHP QD LEDs is shown in Figure S5. The MHP LEDs with DAT exhibited electroluminescence (EL) with an emission peak of $471 \mathrm{~nm}$ and a narrow FWHM of $\sim 17 \mathrm{~nm}$ (Figure $3 \mathbf{b})$. The color coordinate of the LED is $(0.129,0.087)$, which nears the Rec. 2020 specifications for a primary blue emitter. 

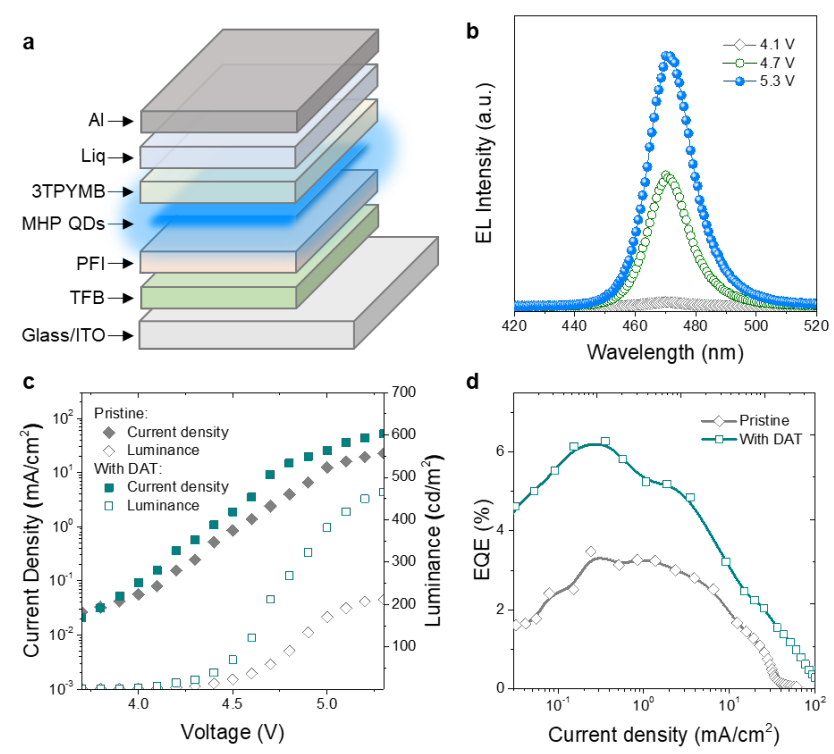

d
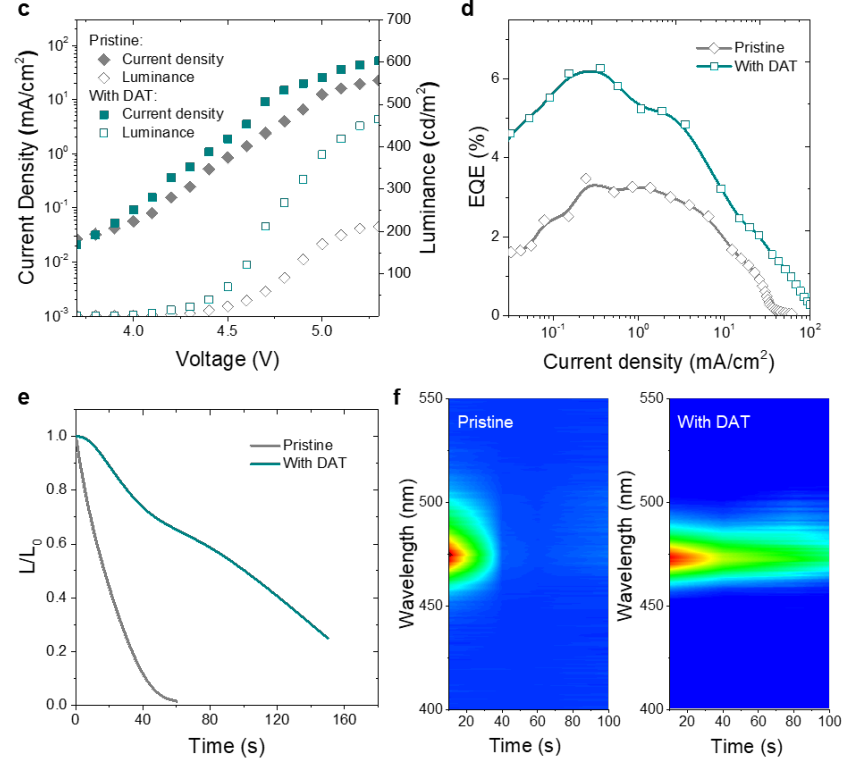

Figure 3. (a) Device structure, (b) normalized EL spectra, (c) Luminance-voltage-current density, (d) EQE-current density, (e) operational lifetimes, and (f) peak position at different operation time for pristine device and the DAT-treated device at a constant voltage bias of $4.5 \mathrm{~V}$.

Figure 3c shows luminance and current density characteristics as a function of applied voltage for pristine device and the DAT-treated device. The maximum luminance for DATtreated device reaches $465 \mathrm{~cd} / \mathrm{cm}^{2}$, over two times higher than the pristine device $\left(210 \mathrm{~cd} / \mathrm{cm}^{2}\right)$. The maximum EQE for DAT-treated device is $6.3 \%$ (Figure 3d), a record for perovskite LEDs with emission between 460-480 nm (Table 1). The EQE is nearly twice as high of that of pristine MHP QD LEDs (3.5\%), which we attribute to the reduced $\mathrm{Cl}$ vacancy density and promoted radiative recombination.

To evaluate the spectral stability, we measured the EL spectra of pristine and DAT-treated devices at different voltages (Figure S6). The pristine MHP device shows increased emission at longer wavelength with increasing voltage bias. In stark contrast to the pristine MHP LEDs, the EL of DATtreated devices only shows a slight shift, indicating that ion migration is more hindered in the devices with DAT. We then measured the operational stability of the MHP devices under a constant voltage bias of $4.5 \mathrm{~V}$. The DAT-treated devices show a half-lifetime close to $99 \mathrm{~s}$, much higher than pristine MHP QD LEDs (17s) (Figure 3e, f). The improved device stability could be ascribed to the suppressed $\mathrm{Cl}$ vacancy density, which suppresses ion migration by reducing the hoping sites. It should be noted, the poor operational stability of blue perovskite LEDs - whether using quantum- tuned pure bromide perovskite (such as $\mathrm{Cs} \mathrm{PbBr}_{3}$ ) or mixed $\mathrm{Br}-\mathrm{Cl}$ perovskite as emitter - is a major challenge for the field, despite the impressive improvements that are being reported lately in the EQEs of these devices. For MHP LEDs, the most obvious reason for the low stability is the ion migration occurring under the bias, which will lead to $\mathrm{Cl}$-rich and $\mathrm{Cl}$-poor phases. In order to enhance the operational stability of MHP LEDs, further research efforts are needed to find suitable passivating agents that also simultaneously act as strong ion migration inhibiters.

In summary, we have demonstrated a facile strategy to passivate $\mathrm{Cl}$ vacancy with non-polar-solvent soluble organic pseudohalides, leading to greatly enhanced efficiency and stability of MHP QD LEDs. As a result of Cl vacancy passivation, MHP QDs treated with DAT exhibit near-unity PLQYs. The Cl-vacancy passivation enabled us to realize efficient blue $(\sim 40 \mathrm{~nm}$, i.e. in the Rec 2020 range relevant display standards) MHP QD LEDs with an EQE of $6.3 \%$ (vs. $3.5 \%$ for pristine devices) and a half-lifetime of $\sim 99 \mathrm{~s}$ (vs. $\sim 17 \mathrm{~s}$ for the pristine devices). This work highlights the crucial role of $\mathrm{Cl}$ vacancy passivation for enhancing the efficiency and stability of MHP QD LEDs, and provides an avenue for significantly improving blue perovskite LEDs relevant to display applications.

\section{ASSOCIATED CONTENT}

\section{Supporting Information}

The Supporting Information is available free of charge on the ACS Publications website at

DOI: 10.XXX.

Experimental details and additional data.

\section{AUTHOR INFORMATION}

\section{Corresponding Author}

*Isliao@suda.edu.cn

Author Contributions

"These authors contributed equally.

\section{Notes}

The authors declare no competing financial interests.

\section{ACKNOWLEDGMENT}

The authors acknowledge the funding support from KAUST, National Natural Science Foundation of China (Nos. 61575136 and 51773141), and Collaborative Innovation Centre of Suzhou Nano Science and Technology (Nano-CIC) by the Priority Academic Program. E.H.S. and all coauthors from the Department of Electrical and Computer Engineering at the University of Toronto acknowledge the financial support from the Ontario Research Fund-Research Excellence Program and from the Natural Sciences and Engineering Research Council of Canada (NSERC). 


\section{REFERENCES}

1. Cao, Y.; Wang, N.; Tian, H.; Guo, J.; Wei, Y.; Chen, H.; Miao, Y.; Zou, W.; Pan, K.; He, Y.; Cao, H.; Ke, Y.; Xu, M.; Wang, Y.; Yang, M.; Du, K.; Fu, Z.; Kong, D.; Dai, D.; Jin, Y.; Li, G.; Li, H.; Peng, Q.; Wang, J.; Huang, W., Perovskite light-emitting diodes based on spontaneously formed submicrometre-scale structures. Nature 2018, 562 (7726), 249-253.

$2 . \quad$ Lin, K.; Xing, J.; Quan, L. N.; de Arquer, F. P. G.; Gong, X.; Lu, J.; Xie, L.; Zhao, W.; Zhang, D.; Yan, C.; Li, W.; Liu, X.; Lu, Y.; Kirman, J.; Sargent, E. H.; Xiong, Q.; Wei, Z., Perovskite lightemitting diodes with external quantum efficiency exceeding 20 per cent. Nature 2018, 562 (7726), 245-248.

3. Chiba, T.; Hayashi, Y.; Ebe, H.; Hoshi, K.; Sato, J.; Sato, S.; Pu, Y.-J.; Ohisa, S.; Kido, J., Anion-exchange red perovskite quantum dots with ammonium iodine salts for highly efficient light-emitting devices. Nat. Photonics 2018, 12 (11), 681-687.

4. Jiang, Y.; Qin, C.; Cui, M.; He, T.; Liu, K.; Huang, Y.; Luo, M.; Zhang, L.; Xu, H.; Li, S.; Wei, J.; Liu, Z.; Wang, H.; Kim, G.-H.; Yuan, M.; Chen, J., Spectra stable blue perovskite light-emitting diodes. Nat. Commun. 2019, 10 (1), 1868.

5. Hou, S.; Gangishetty, M. K.; Quan, Q.; Congreve, D. N., Efficient Blue and White Perovskite Light-Emitting Diodes via Manganese Doping. Joule 2018, 2 (11), 2421-2433.

6. Vashishtha, P.; Ng, M.; Shivarudraiah, S. B.; Halpert, J. E., High Efficiency Blue and Green Light-Emitting Diodes Using Ruddlesden-Popper Inorganic Mixed Halide Perovskites with Butylammonium Interlayers. Chem. Mater. 2019, 31 (1), 83-89. 7. Wang, Q.; Wang, X.; Yang, Z.; Zhou, N.; Deng, Y.; Zhao, J.; Xiao, X.; Rudd, P.; Moran, A.; Yan, Y.; Huang, J., Efficient skyblue perovskite light-emitting diodes via photoluminescence enhancement. Nat. Commun. 2019, 10 (1), 5633.

8. Xing, J.; Zhao, Y.; Askerka, M.; Quan, L. N.; Gong, X.; Zhao, W.; Zhao, J.; Tan, H.; Long, G.; Gao, L.; Yang, Z.; Voznyy, O.; Tang, J.; Lu, Z.-H.; Xiong, Q.; Sargent, E. H., Color-stable highly luminescent sky-blue perovskite light-emitting diodes. Nat. Commun. 2018, 9 (1), 3541.

9. $\quad$ Liu, Y.; Cui, J.; Du, K.; Tian, H.; He, Z.; Zhou, Q.; Yang, Z.; Deng, Y.; Chen, D.; Zuo, X.; Ren, Y.; Wang, L.; Zhu, H.; Zhao, B.; Di, D.; Wang, J.; Friend, R. H.; Jin, Y., Efficient blue light-emitting diodes based on quantum-confined bromide perovskite nanostructures. Nat. Photonics 2019, 13 (11), 760-764.

10. Li, Z.; Chen, Z.; Yang, Y.; Xue, Q.; Yip, H.-L.; Cao, Y., Modulation of recombination zone position for quasi-twodimensional blue perovskite light-emitting diodes with efficiency exceeding 5\%. Nat. Commun. 2019, 10 (1), 1027.

11. Dutta, A.; Behera, R. K.; Pal, P.; Baitalik, S.; Pradhan, N., Near-Unity Photoluminescence Quantum Efficiency for All $\mathrm{CsPbX}_{3}(\mathrm{X}=\mathrm{Cl}, \mathrm{Br}$, and I) Perovskite Nanocrystals: A Generic Synthesis Approach. Angew. Chem., Int. Ed. 2019, 58 (17), 5552-5556.

12. Pradhan, N., Tips and Twists in Making High Photoluminescence Quantum Yield Perovskite Nanocrystals. ACS Energy Lett. 2019, 4 (7), 1634-1638.

13. Zhang, B.-B.; Yuan, S.; Ma, J.-P.; Zhou, Y.; Hou, J.; Chen, X.; Zheng, W.; Shen, H.; Wang, X.-C.; Sun, B.; Bakr, O. M.; Liao, L.S.; Sun, H.-T., General Mild Reaction Creates Highly Luminescent Organic-Ligand-Lacking Halide Perovskite Nanocrystals for Efficient Light-Emitting Diodes. J. Am. Chem. Soc. 2019, 141 (38), 15423-15432.

14. Protesescu, L.; Yakunin, S.; Bodnarchuk, M. I.; Krieg, F.; Caputo, R.; Hendon, C. H.; Yang, R. X.; Walsh, A.; Kovalenko, M. V., Nanocrystals of Cesium Lead Halide Perovskites (CsPbX, $\mathrm{X}=\mathrm{Cl}, \mathrm{Br}$, and I): Novel Optoelectronic Materials Showing
Bright Emission with Wide Color Gamut. Nano Lett. 2015, 15 (6), 3692-3696.

15. Song, J.; Li, J.; Li, X.; Xu, L.; Dong, Y.; Zeng, H., Quantum Dot Light-Emitting Diodes Based on Inorganic Perovskite Cesium Lead Halides $\left(\mathrm{CsPbX}_{3}\right)$. Adv. Mater. 2015, 27 (44), 7162-7167.

16. Pan, J.; Li, X.; Gong, X.; Yin, J.; Zhou, D.; Sinatra, L.; Huang, R.; Liu, J.; Chen, J.; Dursun, I.; El-Zohry, A. M.; Saidaminov, M. I.; Sun, H.-T.; Mohammed, O. F.; Ye, C.; Sargent, E. H.; Bakr, O. M., Halogen Vacancies Enable Ligand-Assisted Self-Assembly of Perovskite Quantum Dots into Nanowires. Angew. Chem., Int. Ed. 2019, 58 (45), 16077-16081.

17. Pan, J.; Shang, Y.; Yin, J.; De Bastiani, M.; Peng, W.; Dursun, I.; Sinatra, L.; El-Zohry, A. M.; Hedhili, M. N.; Emwas, A.H.; Mohammed, O. F.; Ning, Z.; Bakr, O. M., Bidentate LigandPassivated $\mathrm{CsPbI}_{3}$ Perovskite Nanocrystals for Stable NearUnity Photoluminescence Quantum Yield and Efficient Red Light-Emitting Diodes. J. Am. Chem. Soc. 2018, 140 (2), 562565.

18. Krieg, F.; Ochsenbein, S. T.; Yakunin, S.; ten Brinck, S.; Aellen, P.; Süess, A.; Clerc, B.; Guggisberg, D.; Nazarenko, O.; Shynkarenko, Y.; Kumar, S.; Shih, C.-J.; Infante, I.; Kovalenko, M. V., Colloidal CsPbX 3 (X = Cl, Br, I) Nanocrystals 2.0: Zwitterionic Capping Ligands for Improved Durability and Stability. ACS Energy Lett. 2018, 3 (3), 641-646.

19. Nenon, D. P.; Pressler, K.; Kang, J.; Koscher, B. A.; Olshansky, J. H.; Osowiecki, W. T.; Koc, M. A.; Wang, L.-W.; Alivisatos, A. P., Design Principles for Trap-Free $\mathrm{CsPbX}_{3}$ Nanocrystals: Enumerating and Eliminating Surface Halide Vacancies with Softer Lewis Bases. J. Am. Chem. Soc. 2018, 140 (50), 17760-17772.

20. Wu, Y.; Li, X.; Zeng, H., Highly Luminescent and Stable Halide Perovskite Nanocrystals. ACS Energy Lett. 2019, 4 (3), 673-681.

21. Koscher, B. A.; Swabeck, J. K.; Bronstein, N. D.; Alivisatos, A. P., Essentially Trap-Free $\mathrm{CsPbr}_{3}$ Colloidal Nanocrystals by Postsynthetic Thiocyanate Surface Treatment. J. Am. Chem. Soc. 2017, 139 (19), 6566-6569.

22. Ahmed, G. H.; El-Demellawi, J. K.; Yin, J.; Pan, J.; Velusamy, D. B.; Hedhili, M. N.; Alarousu, E.; Bakr, O. M.; Alshareef, H. N.; Mohammed, O. F., Giant Photoluminescence Enhancement in $\mathrm{CsPbCl}_{3}$ Perovskite Nanocrystals by Simultaneous Dual-Surface Passivation. ACS Energy Lett. 2018, 3 (10), 2301-2307.

23. Zheng, X.; Hou, Y.; Sun, H.-T.; Mohammed, O. F.; Sargent, E. H.; Bakr, O. M., Reducing Defects in Halide Perovskite Nanocrystals for Light-Emitting Applications. J. Phys. Chem. Lett. 2019, 10 (10), 2629-2640.

24. Lai, R.; Wu, K., Picosecond electron trapping limits the emissivity of $\mathrm{CsPbCl}_{3}$ perovskite nanocrystals. J. Chem. Phys. 2019, 151 (19), 194701.

$25 . \quad$ Yong, Z.-J.; Guo, S.-Q.; Ma, J.-P.; Zhang, J.-Y.; Li, Z.-Y.; Chen, Y.-M.; Zhang, B.-B.; Zhou, Y.; Shu, J.; Gu, J.-L.; Zheng, L.-R.; Bakr, O. M.; Sun, H.-T., Doping-Enhanced Short-Range Order of Perovskite Nanocrystals for Near-Unity Violet Luminescence Quantum Yield. J. Am. Chem. Soc. 2018, 140 (31), 9942-9951.

26. Gualdrón-Reyes, A. F.; Yoon, S. J.; Barea, E. M.; Agouram, S.; Muñoz-Sanjosé, V.; Meléndez, Á. M.; Niño-Gómez, M. E.; Mora-Seró, I., Controlling the Phase Segregation in Mixed Halide Perovskites through Nanocrystal Size. ACS Energy Lett. 2019, 4 (1), 54-62.

27. Zheng, X.; Hou, Y.; Bao, C.; Yin, J.; Yuan, F.; Huang, Z.; Song, K.; Liu, J.; Troughton, J.; Gasparini, N.; Zhou, C.; Lin, Y.; Xue, D.-J.; Chen, B.; Johnston, A. K.; Wei, N.; Hedhili, M. N.; Wei, M.; Alsalloum, A. Y.; Maity, P.; Turedi, B.; Yang, C.; Baran, D.; 
Anthopoulos, T. D.; Han, Y.; Lu, Z.-H.; Mohammed, O. F.; Gao, F.; Sargent, E. H.; Bakr, O. M., Managing grains and interfaces via ligand anchoring enables $22.3 \%$-efficiency inverted perovskite solar cells. Nat. Energy 2020, DOI: 10.1038/s41560-019-05384.

28. Yassitepe, E.; Yang, Z.; Voznyy, O.; Kim, Y.; Walters, G.; Castañeda, J. A.; Kanjanaboos, P.; Yuan, M.; Gong, X.; Fan, F.; Pan, J.; Hoogland, S.; Comin, R.; Bakr, O. M.; Padilha, L. A.; Nogueira, A. F.; Sargent, E. H., Amine-Free Synthesis of Cesium Lead Halide Perovskite Quantum Dots for Efficient Light-Emitting Diodes. Adv. Funct. Mater. 2016, 26 (47), 8757-8763.

29. Ke, W.; Xiao, C.; Wang, C.; Saparov, B.; Duan, H.-S.; Zhao, D.; Xiao, Z.; Schulz, P.; Harvey, S. P.; Liao, W.; Meng, W.; Yu, Y.; Cimaroli, A. J.; Jiang, C.-S.; Zhu, K.; Al-Jassim, M.; Fang, G.; Mitzi, D. B.; Yan, Y., Employing Lead Thiocyanate Additive to Reduce the Hysteresis and Boost the Fill Factor of Planar Perovskite Solar Cells. Adv. Mater. 2016, 28 (26), 5214-5221.

30. Zheng, X.; Troughton, J.; Gasparini, N.; Lin, Y.; Wei, M.; Hou, Y.; Liu, J.; Song, K.; Chen, Z.; Yang, C.; Turedi, B.; Alsalloum, A. Y.; Pan, J.; Chen, J.; Zhumekenov, A. A.; Anthopoulos, T. D.; Han, Y.; Baran, D.; Mohammed, O. F.; Sargent, E. H.; Bakr, O. M., Quantum Dots Supply Bulk- and Surface-Passivation Agents for Efficient and Stable Perovskite Solar Cells. Joule 2019, 3 (8), 1963-1976.
31. Noel, N. K.; Abate, A.; Stranks, S. D.; Parrott, E. S.; Burlakov, V. M.; Goriely, A.; Snaith, H. J., Enhanced Photoluminescence and Solar Cell Performance via Lewis Base Passivation of Organic-Inorganic Lead Halide Perovskites. ACS Nano 2014, 8 (10), 9815-9821.

32. Yang, S.; Chen, S.; Mosconi, E.; Fang, Y.; Xiao, X.; Wang, C.; Zhou, Y.; Yu, Z.; Zhao, J.; Gao, Y.; De Angelis, F.; Huang, J., Stabilizing halide perovskite surfaces for solar cell operation with wide-bandgap lead oxysalts. Science 2019, 365 (6452), 473-478.

33. Zheng, X.; Chen, B.; Dai, J.; Fang, Y.; Bai, Y.; Lin, Y.; Wei, H.; Zeng, Xiao C.; Huang, J., Defect passivation in hybrid perovskite solar cells using quaternary ammonium halide anions and cations. Nat. Energy 2017, 2, 17102.

34. Tong, J.; Song, Z.; Kim, D. H.; Chen, X.; Chen, C.; Palmstrom, A. F.; Ndione, P. F.; Reese, M. O.; Dunfield, S. P.; Reid, O. G.; Liu, J.; Zhang, F.; Harvey, S. P.; Li, Z.; Christensen, S. T.; Teeter, G.; Zhao, D.; Al-Jassim, M. M.; van Hest, M. F. A. M.; Beard, M. C.; Shaheen, S. E.; Berry, J. J.; Yan, Y.; Zhu, K., Carrier lifetimes of $>1 \mu \mathrm{s}$ in $\mathrm{Sn}-\mathrm{Pb}$ perovskites enable efficient all-perovskite tandem solar cells. Science 2019, 364 (6439), 475-479.

35. $\quad$ Cai, T.; Li, F.; Jiang, Y.; Liu, X.; Xia, X.; Wang, X.; Peng, J.; Wang, L.; Daoud, W. A., In situ inclusion of thiocyanate for highly luminescent and stable $\mathrm{CH}_{3} \mathrm{NH}_{3} \mathrm{PbBr}_{3}$ perovskite nanocrystals. Nanoscale 2019, 11 (3), 1319-1325.

\section{Table of content}

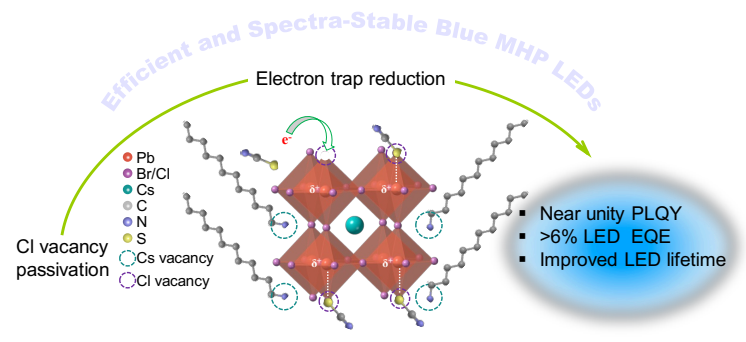

